\title{
Erratum to: High-quality graphene grown directly on stainless steel meshes through CVD process for enhanced current collectors of supercapacitors
}

\author{
NING Jing ${ }^{1,2}$, HAO Long ${ }^{1,2}$, ZHANG XianFeng $^{1}$, LIANG MingHui ${ }^{1} \&$ ZHI LinJie ${ }^{1,3}$ \\ ${ }^{1}$ National Center for Nanoscience and Technology, Beijing 100190, China; \\ ${ }^{2}$ University of Chinese Academy of Sciences, Beijing 100049, China; \\ ${ }^{3}$ School of Materials Science and Engineering, University of Shanghai for Science and Technology, Shanghai 200093, China
}

\begin{abstract}
Erratum to: SCIENCE CHINA Technological Sciences, February 2014 Vol.57 No.2: 259-263 doi: 10.1007/s11431-014-5455-y
\end{abstract}

The first two authors, NING Jing and HAO Long, contributed equally to this work.

The online version of the original article can be found at http://dx.doi.org/10.1007/s11431-014-5455-y 